\title{
El potencial del eye-tracker como herramienta para estudiar el razonamiento matemático: Una experiencia usando videojuegos
}

\author{
Lluís Albarracín ${ }^{1}$ [D, y Aura Hernández-Sabaté ${ }^{2}$ [D
}

\begin{abstract}
Resumen
Los videojuegos son herramientas prometedoras en los entornos educativos ya que tienen características que pueden promover el aprendizaje en un ambiente lúdico. Estudios previos han identificado oportunidades de aprendizaje matemático en un videojuego de estrategia en tiempo real a partir de analizar la interacción de los alumnos al tomar decisiones. Este tipo de análisis presenta dificultades al no poder observar los procesos no verbalizados por los alumnos. En este artículo presentamos una primera herramienta de análisis basada en el eye-tracker para identificar procesos de resolución de problemas matemáticos mientras se juega a un videojuego de estrategia. Los primeros resultados preliminares muestran el potencial de la herramienta desarrollada para identificar procesos de resolución de problemas matemáticos.
\end{abstract}

\section{Abstract}

Video games are promising tools in educational environments as they have characteristics that can promote learning in a playful environment. Previous studies have identified opportunities for mathematical learning in a strategy video game in real time from analyzing the interaction of students when making decisions. This type of analysis presents difficulties in not being able to observe the processes not verbalized by the students. In this article we present a first analysis tool based on the eye-tracker to identify mathematical problem solving processes while playing a strategy video game. The first preliminary results show the potential of the tool developed to identify mathematical problem solving processes.

\section{Palabras clave}

Eye-tracker - Procesamiento de imágenes - Videojuegos - Resolución de problemas

\section{Keywords}

Eye-tracker - Image processing — Videogames - Problem solving

${ }^{1}$ Universitat Autònoma de Barcelona, España; Profesor Serra Húnter

${ }^{2}$ Universitat Autònoma de Barcelona, España; Centre de Visió per Computador, España

*Autor de correspondencia: lluis.albarracin@uab.cat

\section{Introducción}

Los videojuegos aparecen en las últimas décadas del siglo $\mathrm{XX}$ como un producto de entretenimiento para niños. Su aceptación por otros segmentos de población ha ido creciendo con el tiempo, pasando a ser un fenómeno global empujado por la accesibilidad de dispositivos tecnológicos que permiten su uso. Actualmente, los videojuegos se perciben como un producto orientado al entretenimiento que puede ser disfrutado por personas de cualquier edad o condición.

La variedad de géneros en la que se clasifican los videojuegos en la actualidad es realmente amplia. Desde el punto de vista de la Educación Matemática, algunos videojuegos pre- sentan características que les permiten promover actividades que podemos conectar con la actividad matemática o el aprendizaje de las matemáticas (Albarracín, Hernández-Sabaté, y Gorgorió, 2017). Algunos ejemplos pueden ser los videojuegos del género puzzle como Portal 2 (Albarracín, 2013), los videojuegos de gestión deportiva como FX Futbol (Albarracín, 2014a) o los videojuegos de estrategia en tiempo real, como pueden ser Age of Empires o Starcraft (Albarracín, 2014b). Por otro lado, en la literatura científica se pueden encontrar diversas investigaciones que arrojan luz sobre la forma en la que algunos videojuegos promueven determinados aprendizajes matemáticos, pero en muchos casos nos encontramos 
con videojuegos diseñados específicamente para ser usados como actividad de aula. Estos estudios se enmarcan en una corriente que recibe el nombre de serious games, tratando de recoger aquellas iniciativas que trascienden a la naturaleza de los videojuegos como productos para el entretenimiento. En muchos casos estos videojuegos diseñados y desarrollados para el aprendizaje simplifican o desnaturalizan el proceso habitual de juego que proponen los videojuegos comerciales, con lo que se difuminan algunos aspectos clave como la motivación de los alumnos o la complejidad de la actividad de juego.

Por ello, y desde la perspectiva de la Educación Matemática, entendemos que los videojuegos no se han explotado todavía de forma plena como instrumentos educativos. Uno de los principios básicos del diseño de una herramienta educativa es que los objetivos educativos se alineen con los de la actividad propuesta, hecho que no se consigue plenamente al crear un videojuego dirigido a acercar determinados contenidos o procedimientos a los alumnos. En consecuencia los autores nos decantamos por un proceso inverso, en el que se estudian las características de la actividad promovida por determinados videojuegos y se identifican sus potencialidades didácticas. Desde esta perspectiva, en un trabajo exploratorio Hernández-Sabaté, Joanpere, Gorgorió, y Albarracín (2015) se identificaron los procesos de resolución de problemas matemáticos de alumnos entre 10 y 12 años de edad al jugar en parejas a un videojuego de estrategia en tiempo real (RTS) del género Tower defense. En nuestro estudio identificamos que la actividad de los jugadores incluye ciclos de resolución de problemas entrelazados que se forman por procesos de Observación - Planificación - Toma de Decisiones, donde utilizan explícitamente conceptos de contenido numérico y geométrico y de relación funcional entre variables. Una de las dificultades de este estudio se presenta en la forma de observar los elementos del juego que los alumnos consideran antes de tomar una decisión. Por ello, hemos iniciado una línea de investigación usando una herramienta de análisis basada en un eye-tracker, entendiendo que esta tecnología adaptada a las circunstancias del juego debería permitirnos observar elementos de resolución de problemas que no hemos podido identificar a partir de gravaciones genéricas de alumnos jugando.

\section{Antecedentes}

\subsection{Videojuegos como herramientas educativas}

Los videojuegos han demostrado ser una herramienta educativa atractiva por dos aspectos principales. Por una parte, se diseñan a partir de normas y objetivos específicos, con lo que se pueden ajustar a objetivos de aprendizaje concretos. Por otro lado, la naturaleza de la interacción hombre-máquina permite a los jugadores obtener una respuesta inmediata a sus acciones (Dickey, 2005). En una revisión sistemática de la literatura, Connolly, Boyle, MacArthur, Hainey, y Boyle (2012) identificaron aquellos estudios que proporcionan evidencia empírica del impacto positivo de los videojuegos en diferentes aspectos del aprendizaje escolar. En su trabajo encontraron evidencias de mejoras en las habilidades motoras y perceptivas, así como en diversas habilidades cognitivas como la rotación mental, la memoria o la resolución de problemas, esta expresada en un sentido amplio.

En el campo específico de la Educación Matemática existen algunos estudios dirigidos a determinar el impacto del uso de los videojuegos en el aprendizaje de contenidos matemáticos. En la investigación descrita en Rosas y cols. (2003) se utilizaron varios videojuegos que proponen retos y rompecabezas a los estudiantes. Estos videojuegos permiten a los alumnos trabajar directamente con contenidos matemáticos, como son las secuencias numéricas, los problemas de suma y resta, la estimación y el reconocimiento de figuras geométricas. Los resultados de este estudio muestran un aumento en la motivación de los estudiantes durante el trabajo en clase, mostrando también un aumento en la cooperación e interacción verbal entre los estudiantes. Además, los estudiantes participantes mejoraron significativamente sus resultados en una prueba de conocimiento matemático a un nivel más alto que los estudiantes del grupo de control que no usaron videojuegos.

Ke (2008) analiza el comportamiento de los estudiantes que juegan a algunos minijuegos diseñados específicamente para el aprendizaje de las matemáticas. El autor destaca que un aspecto clave del diseño de estos videojuegos es la relación entre los objetivos del juego y los objetivos de aprendizaje y concluye que esta alineación no se manifiesta adecuadamente en la mayoría de videojuegos diseñados específicamente para el aprendizaje de las matemáticas.

En un estudio más reciente, Kiili, Devlin, Perttula, Tuomi, y Lindstedt (2015) utilizaron dos videojuegos en la misma propuesta didáctica con alumnos de los primeros cursos de educación primaria. Los videojuegos utilizados, Semideus y Wuzzit Trouble, han sido diseñados a partir de una representación visual dinámica de determinadas construcciones matemáticas (la línea numérica y la aritmética de los números enteros) para construir un videojuego a su alrededor. De esta manera, para resolver los puzzles y desafíos del juego es necesario entender y resolver el problema matemático que subyace en la situación planteada. Los resultados de este estudio muestran una mejora en los resultados sobre aspectos numéricos entre los alumnos que siguieron la propuesta docente. Mientras tanto, los autores Pope y Mangram (2015) complementan este estudio analizando las estrategias desarrolladas por los estudiantes mientras juegan a Wuzzit Trouble. Estos autores señalaron que el videojuego obliga a los estudiantes a revisar constantemente las estrategias utilizadas, incluso a desarrollar aspectos conceptuales complejos como la factorización de los números naturales y a combinarlos para resolver los desafíos propuestos.

De esta forma, podemos afirmar que el uso de videojuegos orientados al aprendizaje de las matemáticas es un campo de estudio en el que existen posibilidades de progreso que deben considerar la propia naturaleza de los videojuegos así como 
del aprendizaje de las matemáticas.

\subsection{Eye-tracker en el aprendizaje con videojuegos}

Las técnicas de rastreo ocular utilizando un eye-tracker, dispositivo que capta los movimientos oculares de las personas que miran ciertos estímulos (Chen y Yang, 2014), han ganado cada vez más atención y popularidad en la investigación en Educación Matemática en los últimos años (Andrá y cols., 2015). Después de haber sido utilizadas en diversos campos de investigación como la psicología, la psicolingüística, los sistemas visuales, la investigación de mercado y el diseño de productos (Khushaba y cols., 2013; Mele y Federici, 2012). En los últimos años, también ha llegado al campo de los videojuegos como herramienta de interacción con la máquina (Ekman, Poikola, y Mäkäräinen, 2008) o evaluación de experiencias de juego (Yan y El-Nasr, 2006). Potencialmente, el estudio del comportamiento de la mirada puede proporcionar información sobre la atención visual de los jugadores y así ayudar a los diseñadores de juegos a identificar problemas con el juego.

Centrados en el aprendizaje basado en videojuegos, los estudios realizados con eye-trackers no van más allá de la mejora de las interfícies de los juegos educativos mediante la percepción de la experiencia del usuario, sin olvidar su valor para la comunidad educativa. Los autores en Frutos-Pascual, García-Zapirain, y Mehdi (2015) analizaron diferentes habilidades de atención durante la interacción de los usuarios con un conjunto de juegos de puzzle. Concluyeron que los patrones de la mirada les permiten determinar algunas zonas recurrentes donde colocar los contenidos más relevantes. Kinzer y cols. (2012) exploran la comprensión de la historia en los cómics y los videojuegos, descubriendo que la comprensión de la narrativa puede ser mayor en los jugadores que en los lectores de cómics. Kiili, Ketamo, y Kickmeier-Rust (2014) también afirman que el rastreo ocular puede proporcionar información muy profunda y objetiva sobre el diseño del videojuego y la disposición de los elementos para la interacción hombrejuego, aunque la interpretación de los resultados no puede basarse únicamente en los recuentos de fijación y los mapas de puntos relevantes.

En el proceso de interpretación de los datos obtenidos por un eye-tracker aparecen ciertas dificultades. Habitualmente el análisis se lleva a cabo asumiendo la Hipótesis Ojo-mente (Just y Carpenter, 1980) que afirma que los ojos se fijan en lo que la mente está procesando. La Hipótesis Ojo-mente se aplica especialmente a la investigación de los procesos de lectura, donde se desarrolló originalmente, asumiendo que el ojo permanece fijo en una palabra mientras ésta se procesa. Sin embargo, no está claro que esta forma de proceder sea válida para la resolución de problemas matemáticos. Por ejemplo, los estudiantes pueden mirar un punto en el espacio sin registrar el objeto correspondiente o recordar objetos en los que nunca se fijaron (Holmqvist y cols., 2011). De esta forma, el eye-tracker se muestra como una herramienta con una gran potencialidad para el análisis de procesos matemáticos en en- tornos digitales, pero los investigadores deben ser conscientes de sus limitaciones al interpretar los datos obtenidos.

\section{Herramientas externas}

En esta sección se presentan las herramientas externas utilizadas en este trabajo, el videojuego y el eye-tracker.

Vector Tower Defense 2. El videojuego que hemos utilizado en estudios anteriores es Vector Tower Defense 2 (Vector Tower Defense 2, 2019), un videojuego del género de la estrategia en tiempo real (RTS). El objetivo de juego es evitar que las unidades enemigas (llamadas vectoids), que aparecen en oleadas, crucen el mapa. Para conseguirlo, hay que construir torres de defensa para asaltar los vectoids a su paso. Los Vectoids se mueven a lo largo de una ruta específica, y los jugadores tienen una gran variedad de torres, mejoras y opciones de bonificación, lo que les permite explotar al máximo sus habilidades estratégicas. Las consideraciones estratégicas se basan en la elección y colocación de las torres tratando de llevar una gestión óptima de los recursos disponibles. El juego proporciona a los jugadores una gran cantidad de información en pantalla, como son el mapa del juego, la puntuación, los recursos disponibles, los tipos de torres, sus características, su coste y el daño que infligen en cada ataque, entre otros.

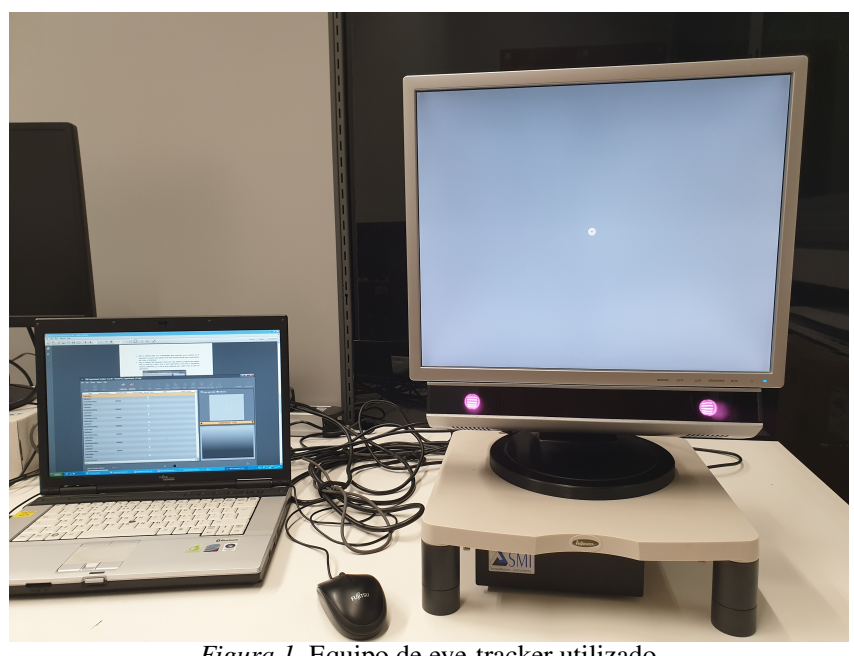

Figura 1. Equipo de eye-tracker utilizado

El dispositivo eye-tracker. El eye-tracker empleado para monitorear las acciones oculares del jugador es el SMI RED 500 de la empresa SensoMotoric Instruments (SMI Vision, 2019). Puede montarse en un monitor de un tamaño que oscile entre 19 y 60 pulgadas y se puede controlar de forma remota desde un PC utilizando el software proporcionado por SMI. Este software de control y adquisición de datos es el SMI iViewX, instalado en un PC que utiliza los datos capturados por los sensores infrarrojos del SMI RED 500. Este software posee calibración manual variable así como modos de calibración automática y rápida. Finalmente, el SMI Begaze es el software utilizado para reproducir, visualizar, analizar y exportar los datos registrados con el eye-tracker, todavía sin 
procesar. El SMI Begaze incluye un editor de Áreas de Interés (AdI) que ayuda a dibujar zonas relevantes en la reproducción del vídeo del seguimiento de la mirada y permite exportar los datos correspondientes a un archivo específico de AdI. La Figura 1 muestra en equipo utilizado, con la pantalla de calibración, los sensores en su parte inferrior y la estación de trabajo que ejecuta los programas necesarios.

\section{EyeMath 1.0: Identificación automática de momentos clave en el juego}

En esta sección presentamos EyeMath 1.0, una herramienta en desarrollo para contribuir a la identificación de oportunidades de aprendizaje matemático al jugar al Vector Tower Defense 2. La Figura 2 muestra el desarrollo del proceso para poder obtener los resultados del análisis.

En primer lugar, el usuario se sienta frente a la pantalla donde jugará al videojuego. Mientras el usuario juega libremente su partida, el eye-tracker registra el seguimiento de su mirada. En especial, registra tres eventos relevantes: las fijaciones (intervalos de tiempo en el que el jugador fija la vista en un punto concreto de la pantalla), las sacadas (movimientos rápidos para desplazar la atención de la mirada a otra zona de la pantalla) y los parpadeos del usuario. Además, el SMI iViewX recoge los datos grabados por el eye-tracker juntamente con las posiciones de los clics del ratón realizados por el usuario durante la partida y el vídeo de la captura de la pantalla de toda la partida. A continuación, se lleva a cabo un proceso automático de los datos recogidos para analizar lo que ocurre en la pantalla y fusionar esa información con las acciones y fijaciones del usuario. El resultado es un nuevo vídeo de la partida con la identificación y resaltado de la zona donde el usuario está mirando en cada momento. Este vídeo permite al investigador la fácil detección de eventos relevantes.

Procesamiento Automático de Datos. Una vez registrados todos los datos relevantes, se inicia el procesamiento automático de datos. En primer lugar, dividimos la pantalla en ocho AdI, basadas en la necesidad de identificar procesos críticos revelados por nuestro estudio anterior (HernándezSabaté y cols., 2015). Estas AdI se muestran en la Figura 3 y son las siguientes: Botón para enviar vectoids, información del juego como vidas, dinero, intereses, bonos, etc., área de selección de las torres, información sobre la torre seleccionada, información de actualización de la torre, información de venta de la torre, información sobre los enemigos actuales y futuros, área de juego.

A partir de la segmentación de la pantalla, creamos una máscara de las AdI y la intersectamos con la información de las fijaciones proporcionada por el eye-tracker. En el caso del área de juego, al ser demasiado grande y estar realmente interesados en saber si el usuario está mirando los vectoids o no, creamos una máscara dinámica de los vectoids (siempre que aparezcan en la pantalla) con técnicas automáticas de detección de objetos (Shaikh, Saeed, y Chaki, 2014).
La Figura 4 muestra el proceso de detección de los vectoids. Se escoge un frame de referencia sin vectoids y el frame actual. A los dos se les aplica una máscara de la ruta que hacen los vectoids. Las imágenes resultantes se restan y se aplica un filtro al resultado para crear la máscara final de los vectoids. Esta máscara dinámica se considera otra AdI, por lo que se añade a la lista de AdIs a considerar.

Finalmente, para cada fotograma, se intersecta la mirada del jugador con el AdI correspondiente. En el caso que mire al área de juego, si el usuario está mirando los vectoids, la máscara resalta los vectoids, pero si el usuario no está mirando los vectoids, sólo se resalta un entorno de la posición de la mirada que ha detectado el eye tracker.

Validación de la herramienta. La máscara dinámica elaborada ha sido validada mediante el etiquetado manual de 3600 fotogramas como 0 o 1 dependiendo de si hay o no vectoids en pantalla y comparándolos con la salida automática correspondiente. Los resultados son un $100 \%$ de fiabilidad. Además, hemos observado los vídeos completos resultantes de 4 partidas de juego y los hemos comparado con los datos originales obteniendo también un $100 \%$ de verdaderos positivos. De esta manera, podemos asegurar que la herramienta es robusta y fiable, de manera que es adecuada para sustentar en el posterior análisis e identificación de oportunidades de aprendizaje matemático, como mostramos en la siguiente sección.

\section{Resultados preliminares}

En esta sección describimos los aspectos cualitativos que EyeMath 1.0 permite identificar en las partidas analizadas. En esta primera aproximación, hemos analizado los videos obtenidos de las partidas de dos jugadores de 13 y 15 años durante 37 y 34 minutos, respectivamente. En ambos casos, grabamos la segunda partida que jugaron, ya que la primera sirvió únicamente para mostrarles las mecánicas del juego. Como describimos en Hernández-Sabaté y cols. (2015), el proceso del Vector Tower Defense 2 se asemeja a una actividad de resolución de problemas matemáticos, debido a los procesos identificados, la complejidad observada y el contenido matemático cubierto. De esta manera, apreciamos los siguientes hallazgos como elementos de resolución de problemas y procesos que pueden ser identificados exclusivamente por medio de EyeMath 1.0.

Datos leídos en la pantalla. Los jugadores leen los datos proporcionados por el juego respecto a las torres y a las características de los enemigos o a otras informaciones relevantes para el juego (vidas restantes o recursos acumulados). La identificación de estos procesos debe permitir observar las diferencias en el juego entre los expertos y los jugadores noveles, así como observar en qué momentos los jugadores expertos necesitan consultar las características de los elementos del juego. Relacionar los momentos en los que los estudiantes consultan estas informaciones permite tener una fuerte intuición de los conocimientos concretos con los que sustentan sus elecciones 


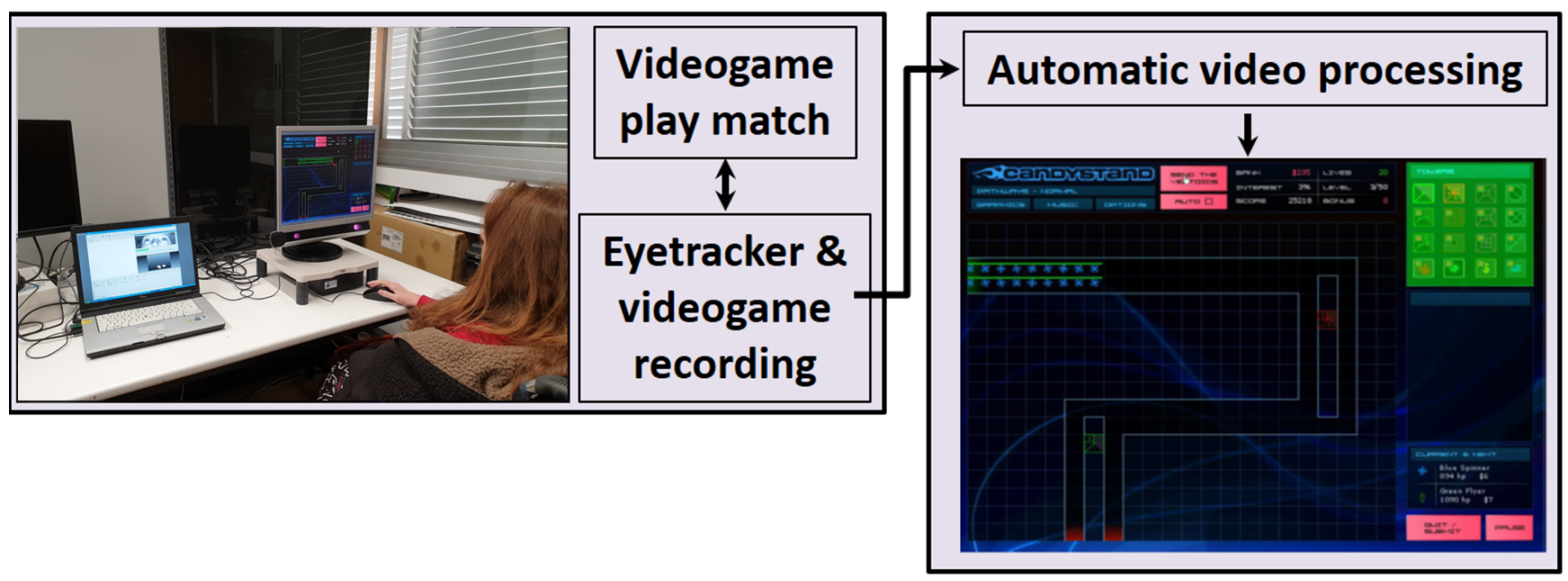

Figura 2. Proceso de la creación del recurso

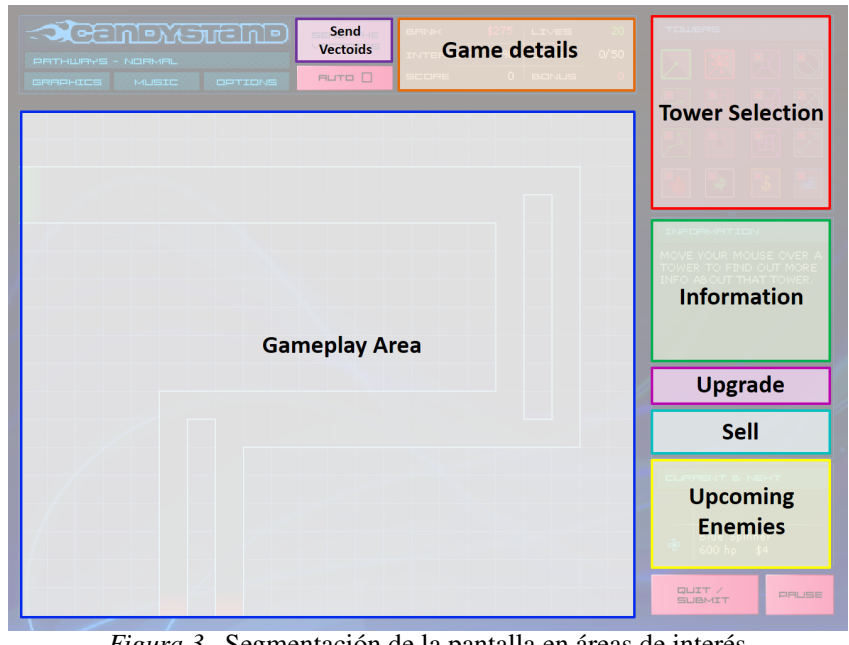

Figura 3. Segmentación de la pantalla en áreas de interés

en el juego, con lo que es una forma de superar algunas de las dificultades conocidas al aplicar la Hipótesis del Ojo-mente (Just y Carpenter, 1980). Además, en muchas ocasiones estos momentos indican el comienzo de un proceso razonado de toma de decisiones y una oportunidad para aprender de los sucesos de juego posteriores.

Colocación de torres y otros elementos. EyeMath 1.0 permite una observación eficaz de las áreas de la pantalla donde un jugador mira cuando está decidiendo la posición donde colocar una torre o usar un elemento de bonificación. En el caso de las torres, los jugadores observan las posibles posiciones en el mapa y el área que cubre la torre para determinar si funcionará correctamente durante el juego. En el caso de los elementos de bonificación, pueden ser colocados en las mismas casillas que las torres y afectar a las torres en una vecindad del elemento de bonificación colocado. El jugador tiene que decidir la posición óptima para exprimir su potencial y la herramienta permite observar en qué aspectos concretos se centra el jugador en el momento en que está tomando la decisión. La Figura 5 muestra un ejemplo de una decisión sobre la colocación de un elemento de bonificación. En ambas imágenes, el jugador está mirando (caja verde) a los bordes del área que cubre el elementos de bonificación para decidir su posición óptima.

El estudio de estas decisiones puede revelar un aspecto clave para entender la toma de decisiones de los estudiantes mientras juegan. Los investigadores hipotizamos que los alumnos crean intuitivamente una función que determina el valor de cada una de las posiciones donde colocar las torres $\mathrm{u}$ otros elementos. Esta nos parece un aspecto relevante para estudiar, ya que relaciona contenidos de medida, procesos de estimación y las relaciones funcionales.

Toma de decisiones en momentos de riesgo. En aquellos momentos en los que se produce una situación opuesta a los intereses del jugador y éste necesita reaccionar con rapidez, la herramienta permite observar claramente las zonas de la pantalla que el jugador consulta para intentar resolver el problema. Esta situación es una clara oportunidad de aprendizaje adicional ya que las acciones tomadas en estas circunstancias no pueden ser apoyadas en un razonamiento lento, dado el corto margen de tiempo, pero pueden ser estudiadas matemáticamente más tarde.

Procesos de anticipación. Observamos que los jugadores se anticipan a las acciones del juego en algunas circunstancias y con objetivos diferentes. Desde el punto de vista del aprendizaje de las matemáticas, este avance denota la necesidad de conseguir datos para analizar la situación del juego y se puede conectar con procesos metacognitivos que, por su naturaleza, son difíciles de estudiar. Hemos observado como los jugadores miran detalladamente áreas de la pantalla donde todavía no han colocado ninguna torre, aunque no colocan ninguna torre inmediatamente después de esta fijación. Entendemos que evalúan las posibilidades de colocarlas en fases futuras 


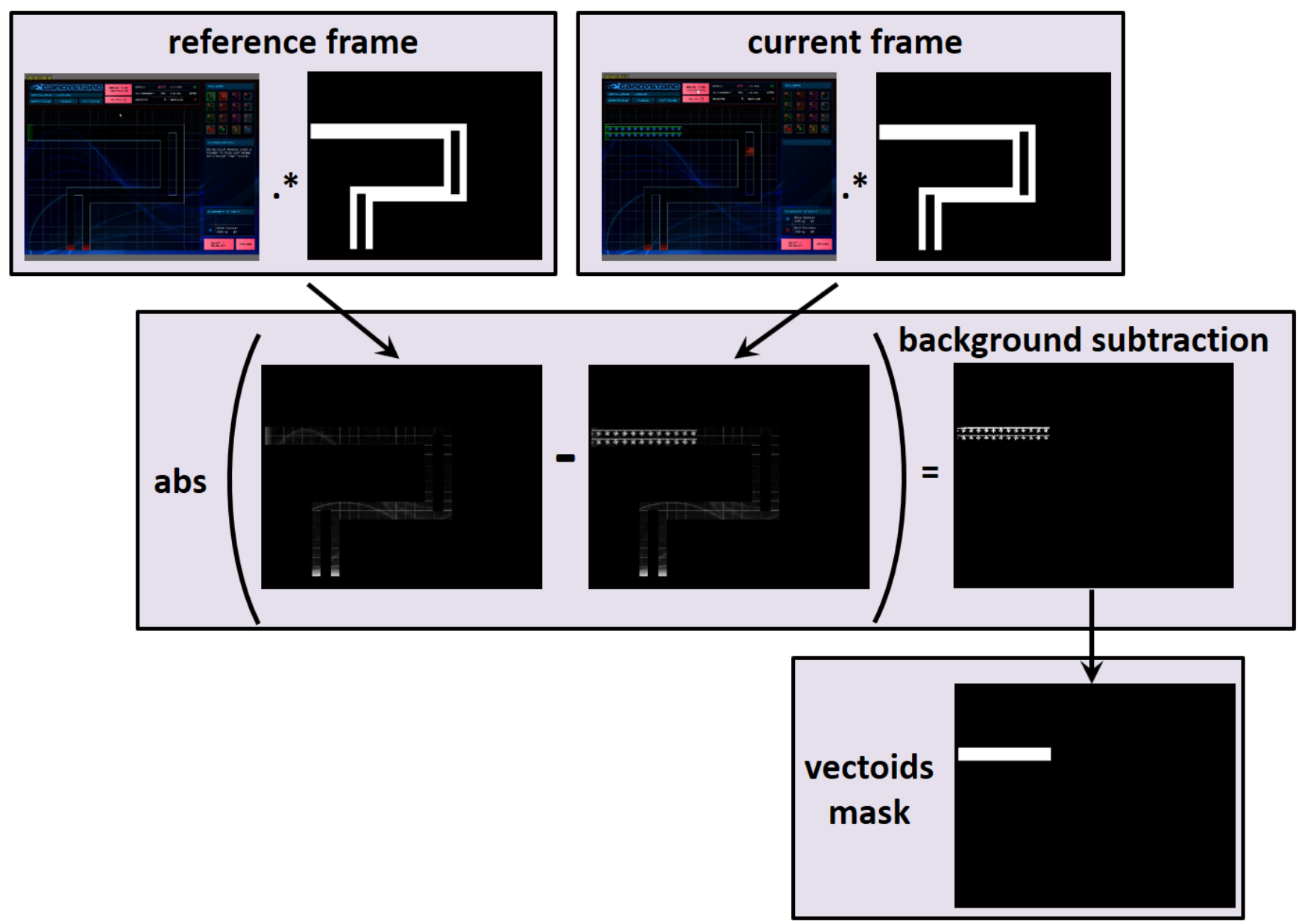

Figura 4. Proceso de detección de los vectoids
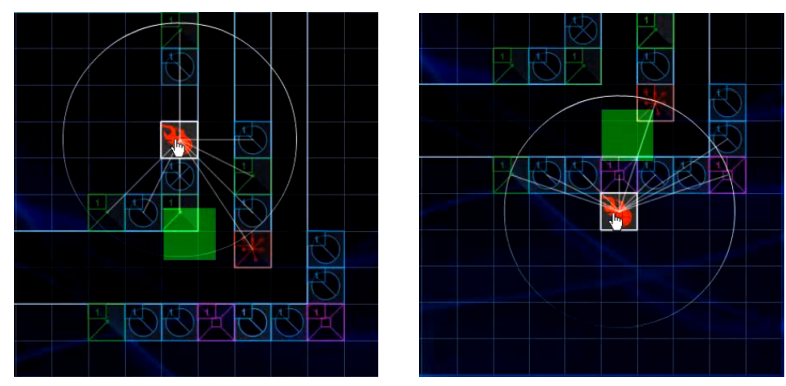

Figura 5. Ejemplo de elección de ubicación de un elemento

del juego. También podemos observar como los jugadores siguen a los vectoids a medida que avanzan en la pantalla pero desvían rápidamente la mirada a las posiciones que los vectoids ocuparán en un futuro inmediato para estimar sobre la marcha si las torres colocadas serán suficiente para detenerlos.

Dadas las características del juego, cada tipo de enemigo se ve afectado de forma diferente por los diferentes tipos de torre. Por lo tanto, las posibilidades de destruir a los enemigos son sustancialmente diferentes en cada ronda. Hemos observado que los jugadores novatos no siguen con la mirada el movimiento de los vectoids en las primeras rondas, pero lo empiezan a hace tan pronto como observan estas dificulta- des. Así, los jugadores aprenden intuitivamente que el poder de las torres depende de sus características según las de los enemigos y necesitan consultarlas en pantalla para sustentar futuras decisiones. La Figura 6 muestra un ejemplo de un proceso de anticipación. La imagen de la izquierda muestra que el jugador está siguiendo los vectoids, mientras que la imagen de la derecha, que se produce 10 segundos después, muestra que el jugador está mirando a otra área del camino donde los vectoids todavía tienen que llegar, ya que considera que existe un peligro potencial de que las torres no detengan a los enemigos y tenga que reaccionar. La reacción que efectue el jugador en estas situaciones puede revelar el conocimiento concreto que posee sobre los elementos del juego, que en gran parte incluye una descripción cuantitativa de estos elementos.

\section{Conclusiones y trabajo futuro}

En este artículo presentamos una herramienta de análisis, el EyeMath 1.0, que permite identificar aspectos de resolución de problemas matemáticos en un videojuego de estrategia a partir del uso de un eye-tracker. En particular, hemos mostrado su potencial destacando, no sólo algunos aspectos previamente identificados en un trabajo anterior Hernández-Sabaté y cols. (2015), sino también algunos otros que difícilmente serán ex- 

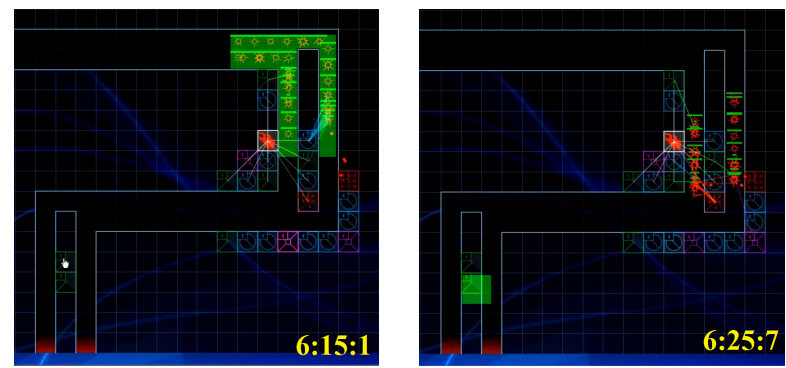

Figura 6. Ejemplo de un proceso de anticipación

presados verbalmente por los jugadores, como la información que usan para sustentar sus decisiones o el modelo mental en forma de función con el que valoran cada posición en el mapa de juego. En consecuencia, el uso de un eye-tracker abre un abanico de posibilidades para analizar procesos mentales de toma de decisiones que involucran conocimientos matemáticos que hasta ahora no se han podido analizar.

En particular, el EyeMath 1.0 permite identificar los procesos de juego, como la forma en que los usuarios interpretan los datos de la pantalla en forma de procesos de resolución de problemas matemáticos, como la forma en que los usuarios colocan las torres en el campo, y los metacognitivos, como la anticipación de procesos. Todos ellos deben ser explorados en profundidad, ya que este potencial abre la puerta para comprender con más detalle la relación entre la actividad lúdica y el contenido matemático abordado. Además, debería ser un apoyo clave para diseñar actividades para el aula con el objetivo de impulsar el trabajo matemático y sugerir secuencias de actividades que permitan superar propuestas que hemos desarrollado en el pasado (Joanpere, Nicolich, Vila, y Albarracín, 2016) para el videojuego estudiado en este estudio, pero también para poder crear guías concretas de actuación para el profesorado para aprovechar la potencialidad de los videojuegos como herramientas para promover aprendizajes matemáticos (Albarracín, 2019), en especial en procesos que presentan grandes dificultades para los alumnos, como son la resolución de problemas o la modelización matemáticas, competencias que se muestran como clave para el desarrollo personal de los alumnos.

\section{Agradecimientos}

Lluís Albarracín es miembro del grupo de investigación Laboratori Competència Matemàtica en Context (LABCOMeC) 2017 SGR 427.

\section{Referencias}

Albarracín, L. (2013). Portal 2. Suma: Revista sobre Enseñanza y Aprendizaje de las Matemáticas(74), 77-82.

Albarracín, L. (2014a). Los videojuegos de gestión deportiva: Fx fútbol 2.0. Suma: Revista sobre Enseñanza y Aprendizaje de las Matemáticas(76), 75-82.
Albarracín, L. (2014b). Videojuegos de estrategia en tiempo real. Suma: Revista sobre Enseñanza y Aprendizaje de las Matemáticas(75), 77-83.

Albarracín, L., Hernández-Sabaté, A., y Gorgorió, N. (2017). Los videojuegos como objeto de investigación incipiente en educación matemática. Modelling in Science Education and Learning, 10(1), 53-72.

Albarracín, L. (2019). Una guía práctica para el uso de videojuegos en el aula de matemáticas. Epsilon, 101, 101-119.

Andrá, C., Lindström, P., Arzarello, F., Holmqvist, K., Robutti, O., y Sabena, C. (2015). Reading mathematics representations: An eye-tracking study. International Journal of Science and Mathematics Education, 13(2), 237-259.

Chen, Y.-C., y Yang, F.-Y. (2014). Probing the relationship between process of spatial problems solving and science learning: An eye tracking approach. International Journal of Science and Mathematics Education, 12(3), 579-603.

Connolly, T. M., Boyle, E. A., MacArthur, E., Hainey, T., y Boyle, J. M. (2012). A systematic literature review of empirical evidence on computer games and serious games. Computers \& Education, 59(2), 661-686.

Dickey, M. D. (2005). Engaging by design: How engagement strategies in popular computer and video games can inform instructional design. Educational Technology Research and Development, 53(2), 67-83.

Ekman, I. M., Poikola, A. W., y Mäkäräinen, M. K. (2008). Invisible eni: using gaze and pupil size to control a game. En Chi'08 extended abstracts on human factors in computing systems (pp. 3135-3140).

Frutos-Pascual, M., García-Zapirain, B., y Mehdi, Q. H. (2015). Where do they look at? analysis of gaze interaction in children while playing a puzzle game. En Computer games: Ai, animation, mobile, multimedia, educational and serious games (cgames), 2015 (pp. 103-106).

Hernández-Sabaté, A., Joanpere, M., Gorgorió, N., y Albarracín, L. (2015). Mathematics learning opportunities when playing a tower defense game. International Journal of Serious Games, 2(4), 57-71.

Holmqvist, K., Nyström, M., Andersson, R., Dewhurst, R., Jarodzka, H., y Van de Weijer, J. (2011). Eye tracking: A comprehensive guide to methods and measures. OUP Oxford.

Joanpere, M., Nicolich, M., Vila, M., y Albarracín, L. (2016). Un videojuego de estrategia para proponer problemas de matemáticas. Aula de innovación educativa(248), 24-28.

Just, M. A., y Carpenter, P. A. (1980). A theory of reading: From eye fixations to comprehension. Psychological Review, 87(4), 329-354.

Ke, F. (2008). A case study of computer gaming for math: Engaged learning from gameplay? Computers \& Edu- 
cation, 51(4), 1609-1620.

Khushaba, R. N., Wise, C., Kodagoda, S., Louviere, J., Kahn, B. E., y Townsend, C. (2013). Consumer neuroscience: Assessing the brain response to marketing stimuli using electroencephalogram (eeg) and eye tracking. Expert Systems with Applications, 40(9), 3803-3812.

Kiili, K., Devlin, K., Perttula, T., Tuomi, P., y Lindstedt, A. (2015). Using video games to combine learning and assessment in mathematics education. International Journal of Serious Games, 2(4).

Kiili, K., Ketamo, H., y Kickmeier-Rust, M. D. (2014). Eye tracking in game-based learning research and game design. International Journal of Serious Games, 1(2).

Kinzer, C. K., Turkay, S., Hoffman, D. L., Gunbas, N., Chantes, P., Chaiwinij, A., y Dvorkin, T. (2012). Examining the effects of text and images on story comprehension: An eye-tracking study of reading in a video game and comic book. 61st Yearbook of the Literacy Research Association, 259-275.

Mele, M. L., y Federici, S. (2012). Gaze and eye-tracking solutions for psychological research. Cognitive processing, 13(1), 261-265.

Pope, H., y Mangram, C. (2015). Wuzzit trouble: The influence of a digital math game on student number sense. International Journal of Serious Games, 2(4).

Rosas, R., Nussbaum, M., Cumsille, P., Marianov, V., Correa, M., Flores, P., ... others (2003). Beyond nintendo: design and assessment of educational video games for first and second grade students. Computers \& Education, 40(1), 71-94.

Shaikh, S. H., Saeed, K., y Chaki, N. (2014). Moving object detection using background subtraction. En Moving object detection using background subtraction (pp. 1523). Springer.

SMI Vision. (2019). Descargado de https: / / imotions - com/hardware/smi-red500/

Vector Tower Defense 2 . (2019). Descargado de http://www.arcadeboss.com/ game-1823-9-Vector-TD-2.html

Yan, S., y El-Nasr, M. S. (2006). Visual attention in 3d video games. En Proceedings of the 2006 symposium on eye tracking research \& applications (pp. 42-42). 